\title{
Editors Note: Journal of Gerontology and Geriatric Research
}

Rajesh Chauhan*

Family Healthcare Centre, India

*Corresponding author: Rajesh Chauhan, Family Healthcare Centre, 154 / 6-B, Avas Vikas Colony Sikandra, AGRA- 282007, India, Tel: +91-9760021192; E-mail: drrajeshchauhan@gmail.com

Rec Date: Sep 18, 2016, Acc Date: Sep 20, 2016, Pub Date: Sep 22, 2016

Copyright: $\odot 2016$ Chauhan $\mathrm{R}$, et al. This is an open-access article distributed under the terms of the Creative Commons Attribution License, which permits unrestricted use, distribution, and reproduction in any medium, provided the original author and source are credited.

\section{Editor's Note}

All around the world geriatric population is increasing. This is accompanied by its unique problems including issues of senescence and frailty, and at times complicated by multi-morbidities. For a graceful ageing it's about time that we start to explore meaningful newer ideas, concepts and research that is aimed towards helping geriatric population. Journal of Gerontology and Geriatric Research is perhaps doing a yeoman's service for the mankind and for the geriatric research. Our journal has come a long way from its inception, and from its first online open access issue in March 2012.

The current issue has a bouquet of interesting original peer reviewed researches, short communications, commentaries, review articles, case reports, and letters to editor. The views expressed are of the authors, and as an editor it is my privilege to guide you through this latest edition of our journal. I would like to thank all the authors for their nice contributions, and wish them the very best. To read the complete article, please click on the links below.

\section{Geriatric Depression and Cognitive Impairment: A Follow up Study}

As such to diagnose depression in the aged is not that easy. This elaborate study from Argentina by Dillon C, Filipin F, Taragano FE, Heisecke S, Camelo JL, et al, gives us different clinical modalities of diagnosing depression and highlights some of the most common causes of depression in the elderly. They have considered the medical, psychiatric and sociological risk factors for depression, and have done a follow up of their patients at 6 months. Their take home message is that it is important to implement health policies to inform the community, prevent associated risk factors, and promote appropriate treatments and rehabilitation.

\section{The Benefit and Tolerability of Adjuvant Chemotherapy in Elderly Stage III Colon Cancer Patients: A 3 Year Retrospective Audit}

Colon cancer is quite a frequent morbidity that affects the elderly. This study by Lin HS, Turner DJ, Srivastava A, Jameson MB is about adjuvant chemotherapy amongst elderly patients of stage III colon cancer who were identified retrospectively from three years oncology database of the Regional Cancer Centre at Waikato Hospital, New Zealand. The aim of this study was to evaluate the merit of adding oxaliplatin to fluoropyrimidine-based adjuvant chemotherapy in older adults with colon cancer. They have done a thorough review of the treatment, and the associated benefits that possibly have been because of the adjuvant chemotherapy.

\section{Combined Ultrasound and Electric Field Stimulation Aids the Healing of Chronic Pressure Ulcers}

Chronic pressure ulcers is a fairly common morbidity in the elderly, and it is quite difficult to manage pressure ulcers. Rosenblum $J$ and Papamichael $M$ from the Shaarei Zedek Medical Center, Jerusalem, Israel, have come up with a novel method of chronic pressure ulcers. They have used combined ultrasound and electric field stimulation aids, and their results speak out for their technique, as is shown in the figures below.

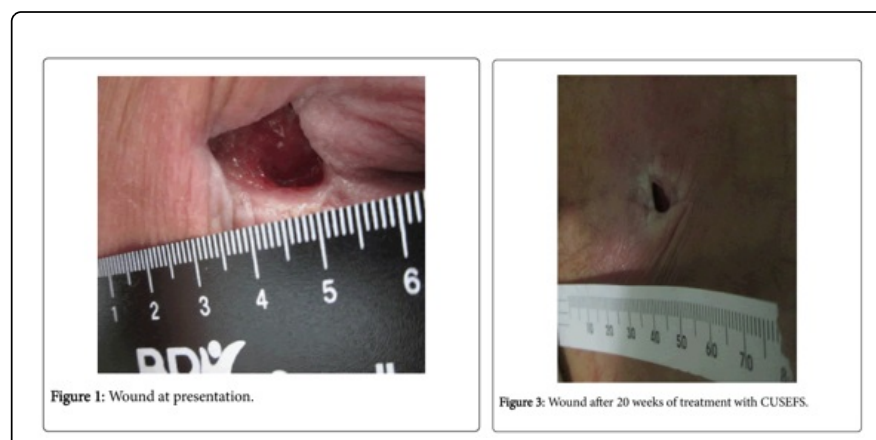

Figure 1: Wound at Presentation.

\section{Food Security among the Elderly: An Area of Concern}

In this short communication Paramita Sengupta from CMC Ludhiana, India has expressed concern about food security among the elderly. Going by the estimates put up by US Census Bureau, the current elderly population is going to get doubled by the year 2050, and this would surely raise concerns about the food security. The author has considered the four pillars of food security, which are availability, access, utilization, and stability, and has gone ahead to identify the four components of food insecurity in the elderly. While emphasizing the household food security survey module (HFSSM) as a reasonable way to assess food insecurity in older adults, the author has identified some of the associated problems.

\section{Comparing the Tools to Identify Potentially Inappropriate Medications in the Elderly and Future Research Directions}

In their commentary about this potentially inappropriate medications problem in elderly the authors, Brown JD, Hutchison LC, and Martin BC, have compared certain tools like for example the Beers and STOPP Criteria that could prevent inappropriate medications in elderly patients of USA. They have expressed the need of continued 
updates and refinements so as to include new evidence and account for newly marketed medications.

\section{The Perception of Special Needs Dentistry amongst General Dentists within Western Australia, Australia}

Derbi HA and Borromeo GL from Australia have identified from their research that special needs patients have a higher prevalence of oral disease and greater unmet dental needs compared to the general population, and that a number of oral health issues are related to both their disability and complex health needs that can in many instances prevent them from receiving appropriate dental care. In this research they have included a large number of dentists, and have sought to seek out their perception of special needs dentistry. They have concluded that lack of dentist skills and knowledge in managing people with special needs were one of the most frequent barriers, and that it can be improved with professional developmental training programs in special needs dentistry.

\section{An Experimental Group Session for Cross-Cultural Social Skills Learning for International Students in Japan}

Tomoko Tanaka and Minako Nakashima from Okayama University, Japan have developed an experimental group session model for improving cross-cultural social skills in international students. They had hypothesized two effects of the session, both short term and long term. During this research, they observed a growth of understanding and acquisition of social skills and a reduction of anxiety in the participants. As per them, this "short-term effects" is assumed to have three dimensions: behavior, cognition, and feeling. They are of the view that by gaining behavioral skills, and the resultant cognitive and emotional changes over a long term would gradually contribute to cross-cultural adaptation in individuals. As per them, behavior learning possibly works favorably in interpersonal and group relationships over long periods. Such a complicated and expanded influence is called the "long-term effect".

\section{Deceased Donor Kidney Transplantation in Patients Aged 70 and Older: Is 70 the New 50?}

Faroo U, Al-Shraideh Y, Farney AC, Palanisamy A, Rogers J, et al, from Wake Forest School of Medicine, Winston-Salem, USA, have found from their research conducted from a retrospective chart review of all deceased donor kidney transplantation that it can be beneficial to an elderly who is 70 year old or more to undergo kidney transplantation. Such results can possibly lead to decrease in morbidity associated with poorly functional kidneys in elderly who are beyond 70 year of age. According to them, with pre-transplant screening and selection, and a robust immunosuppressant protocol and intensive post-transplant surveillance ensures enhanced quality of life and life expectancy.

\section{Oral Dryness in Post Menoapusal Women: Hormonal Impact}

In this review article, Singh B, Kaur K, Narang RS, Singh S, and Kaur P, from India have found that oral dryness and burning mouth is a frequent problem in post menopausal women. They have reviewed the problem and the available modalities of management of oral dryness in quite details. They are of the opinion that oral changes following menopause must be diagnosed properly, so that patients can receive appropriate care.

\section{The Rules in Institutional Care: An Ethnographic Style Study Introduction}

This research article by Angela Kydd, Deidre Wild and Ann Fleming from Department of Nursing, University of the West of Scotland, UK, deals with the four types of rules that a hospital usually has, and their impact on the hospitalized elderly and frail patients. These rules have been identified by them as procedural rules, hospital rules, certain unspoken rules, and spontaneous rules, and have given out their impact on elderly patients. Finally they have made some nice recommendations for improvement in institutional care settings.

\section{Amyands Hernia: An Unexpected Finding during Inguinal Hernia Repair}

This case report of a rare condition wherein the vermiform appendix was found in the hernia sac of an inguinal hernia, has been reported by Vinita Angeline Rajadurai from Australia. Such a condition is termed as Amyands hernia. The author has also reviewed the available literature on the subject.

\section{Nutritional Influences on Age-Related Frailty}

This article reviews extensively about nutritional influences on agerelated frailty. The authors Bair MJ, Chen $\mathrm{HL}$, Wu CH, Lee YK, Lin IT, et al, from Taiwan have reviewed the existing literature on nutritional influences on frailty. They have made specific recommendations about improving intake of macronutrients as well as micronutrients in order to limit frailty in the elderly.

\section{Groves Elder Life (GEL) Project: Virtual Family Visits for Elderly Patients}

This short communication by the authors Khalid Enver, Laura Burns and Ali Enver, from Canada mentions about Groves Memorial Community Hospital in Fergus, Ontario, that had launched the Groves elder life (GEL) project in December 2014. In ths project virtual family visits for in-patient elderly patients were organized with the use of an iPad, skype, etc, and according to the authors the response and feedback was good.

\section{The Publication of Data on Trajectories of Depressive Symptoms in Older Adults and Risk of Dementia Gives Rise to Broadening this Issue on Problems of Patient's Overall Life Trajectories}

Andrzej Brodziak, Alicja Różyk-Myrta and Agnieszka Wolińska, the authors from Poland, have emphasized upon the concept of health trajectories. In that if overall life trajectories of geriatric patients is considered and taken into account, the impact that all the life events may have or contribute towards depressive symptoms in elderly and their correlation with dementia can be discerned easily. This might help to identify and in instituting an early and appropriate management so as to thwart off some of the adverse consequences. 


\section{Long Term Care in Japan and Sweden: A Comparison of Target Population, Needs and Services Provided from 2000-2010}

These authors from Sweden and Japan, Marten Lagergren, Noriko Kurube and Yasuhiko Saito, have compared the effects of long term care needs and the plans that are in vogue in both these countries. It goes without saying that much still needs to be done for the ageing population, and these two countries, Sweden and Japan, are the forerunners perhaps in establishing long term care for the elderly. The authors have carried out a painstaking comparative research and have come up with conclusions and recommendations, which could be highly beneficial for other countries and for the healthcare providers for the elderly.

\section{TeleHealth and Accelerated Aging in the World Population: Future Research Focus}

This letter to editor from Canada by Sema K Aydede essentially deals with two-fold problems of accelerated aging in the world population, and the problem of multi-morbidities. The author has reviewed the present TeleHealth system, and has given some nice recommendations for improving TeleHealth system and in making it more robust, more responsive, and more effective.

\section{Treatment of Dementia in Parkinsonian Patients}

Dementia as such is problematic, but it may be more distressing if it comes on in patients with Parkinsonism. This mini review of a common problem of dementia in Parkinsonism patients, and the challenging problem of its treatment is an interesting read. The authors, Felix-Martin Werner and Rafael Coveñas, from Germany and Spain respectively, have described the pathophysiology, the pathways involved, and the treatment update.

\section{Glucose Role in Treatment of Alzheimers' Disease}

This commentary on the plausible role of glucose in the treatment of Alzheimer's disease by Amos Gelbard from Israel, is an interesting read. The author looks at the role of cortisol as a stress hormone, and its role in raising blood glucose levels when circulating glucose levels go down. According to the author and with a reference provided, this raised cortisol level in patients with Alzheimer's disease leads to abnormal amyloid build up in brain. The author ponders if in first place glucose levels are not allowed to fall below the required threshold, thereby preventing release cortisol and its build up. He is of the view that further research of the impact of glucose on Alzheimer's disease must be undertaken.

\section{Ageing...Global Crisis for Poor Oral Health!}

Radical changes in approaches dental professionals, policymakers, and social understanding about geriatric dentistry is obligatory. This is what Anil Patil, Aniruddha Varekar, Pranav Patil and Anand Shigli from Bharati Vidyapeeth Deemed University Dental College and Hospital, Maharashtra, India, have said. In this commentary, they have analyzed the present scenario of oral health in geriatric population, identified the lacunae, and have given some good recommendations for improving geriatric dental care.

\section{Spousal Relation in Post-Retirement Life}

Harasankar Adhikari from Kolkata, India, in his letter to editor has brought out the oft neglected issue of the spousal relations in postretirement life. Besides other issues, he has also stated that lack in balancing relationship, supporting partner interests, calibrating time together and resolving difficult issues were their prime obstacle of happiness in their elderly stage. Perhaps there is a need to look more deeply in post-retirement life and its effect and impact on spousal relations, and find ways to rejuvenate and harmonize spousal relationship in the elderly. 